\title{
Effects of Steeping Temperature and Time on the Parboiling Efficiency and the Milling Quality of the Parboiled Paddy Rice
}

\author{
Unuigbe Odion Michael ${ }^{1}$, Izuagie Festus Iwarhue ${ }^{2}$, Olawepo Babatunde Bamidele ${ }^{3}$ \\ ${ }^{1}$ Department of Agricultural and Bio-Environmental Engineering Technology, Auchi Polytechnic, Auchi, Nigeria \\ ${ }^{2}$ Department of Electrical/Electronic Engineering Technology, Auchi Polytechnic, Auchi, Nigeria \\ ${ }^{3}$ Department of Mechanical Engineering Technology, Auchi Polytechnic, Auchi, Nigeria
}

Email address:

kamyuf@gmail.com (Unuigbe O. M.)

\section{To cite this article:}

Unuigbe Odion Michael, Izuagie Festus Iwarhue, Olawepo Babatunde Bamidele. Effects of Steeping Temperature and Time on the Parboiling Efficiency and the Milling Quality of the Parboiled Paddy Rice. American Journal of Mechanics and Applications. Vol. 6, No. 1, 2018, pp. 12-16. doi: 10.11648/j.ajma.20180601.13

Received: December 27, 2017; Accepted: January 5, 2018; Published: February 26, 2018

\begin{abstract}
Paddy rice Parboiling is a major processing operation in rice processing. In this study, the effects of steeping temperature and time on the parboiling efficiency and the milling quality of the parboiled paddy rice was determined in the department of agricultural and bio-environmental engineering department was evaluated. During the investigation, the experiment was considered as a $4 \times 3 \times 3$ factorial design, with four levels of steeping temperature $\left(60,65,70\right.$ and $\left.75^{\circ} \mathrm{C}\right)$, three levels of steeping time (2, 3 and 4 hours) and three replications in a Randomized Complete Block Design (RCBD) with steeping time as the blocking factor in order to determine the parboiling efficiency of the machine and milling quality of the parboiled rice grains. The optimum parboiling efficiency of $99.7 \%$ and corresponding milling quality of $98.5 \%$ was obtained at $70^{\circ} \mathrm{C}$ steeping temperature and 3hours steeping time. The data obtained was statically analyzed using SPSS 21.0 software and the result of the analysis shows that the steeping temperature and time has a significant effect on the parboiling efficiency of the machine and the milling quality of the rice grains at $5 \%$ confidence level.
\end{abstract}

Keywords: Parboiler, Temperature, Time, Paddy Rice, Milling Quality

\section{Introduction}

Parboiled rice (also called converted rice) is rice that has been partially boiled in the husk. The three basic steps of parboiling are soaking, steaming and drying (Ndindeng et al., 2014). These steps also make rice easier to process by hand, boost its nutritional profile and change its texture. About $50 \%$ of the world's paddy production is parboiled. The treatment is practiced in many parts of the world such as India, Bangladesh, Pakistan, Myanmar, Malaysia, Nepal, Sri Lanka, Guinea, South Africa, Italy, Spain, Nigeria, Thailand, Switzerland, USA and France (Demont et al., 2012; Ndindeng et al., 2014). Parboiling drives nutrients, especially thiamin, from the bran to the endosperm, hence parboiled white rice is $80 \%$ nutritionally similar to brown rice. Because of this, parboiling was adopted by North American rice growers in the early 20th century (Futakuchi et al., 2013).
Rice parboiling if properly carried out, improves significantly the physical, eating and nutritional quality of the milled rice compared to the non-parboiled counterpart (Manful et al. 2009; Odenigbo et al., 2013). Rice parboiling thus provides an opportunity for the transformation of poor quality paddy from farmer's fields that may be due to changing climatic conditions and poor farmer-miller practices in Nigeria to good quality milled rice (Ndindeng et al., 2014). Parboiled rice is rice that has its starch partially gelatinized by soaking paddy rice or brown rice in water followed by steaming and a drying process. Parboiling increases grain translucency and decreases chalkiness due to starch pre-gelatinization (Bhattacharya, 2008). It also increases grain hardness and manages to reduce grain breakage as a result of the swelling of the starchy endosperm during gelatinization, which heals the preexisting defects (Rao and Juliano, 2011; Bhattacharya, 2008). Milled parboiled rice has also been reported to have a 
lower glycemic index, higher resistant starch content, and higher contents of B-vitamins than milled non-parboiled rice (Odenigbo et al., 2013). In addition, parboiled rice has been shown to possess some unique cooking, flavor, and textural characteristics that are appealing to certain groups of consumers (Heinemann et al., 2006; Prom-U-thai et al., 2010; Demont et al., 2012).

The traditional parboiling process involves soaking rough rice overnight or longer in water at ambient temperature, followed by boiling or steaming the steeped rice at $100^{\circ} \mathrm{C}$ to gelatinize the starch, while the grain expands until the hull's lemma and palea start to separate (Gariboldi, 1984). The parboiled rice is then cooled and sun-dried before storage or milling.

Modern methods involve the use of a hot-water soak at $60^{\circ} \mathrm{C}$ (below the starch gelatinization temperature) for a few hours to reduce the incidence of aflatoxin contamination during the soaking step. Leaching of nutrients during soaking aggravates the contamination, with the practice of recycling the soak water. Soaking sound, rough rice in water inoculated with Aspergillus parasiticus did not result in aflatoxin contamination of parboiled rice, Eliasson, (2000) suggesting that contamination probably has to be present in the grain prior to soaking.

\section{Materials and Methods}

\subsection{Description of the Parboiler Used for the Study}

The parboiler used for the study (Figure 1) comprises of paddy rice and water compartments separated with a thick plate of $2 \mathrm{~mm}$. the perboiler is made up of a temperature regulating device which controls the steeping temperature. During operation of the parboiler, clean water was introduced into the parboiler and preheated to the required temperature before washed paddy rice were weighed and transferred in to the machine. The temperature regulating device is made up of a thermostat and water thermocouple sensor. The thermocouple sensor signal the thermostat once the temperature reaches the desired value and it is displayed on the digital screen of the thermostat, the thermostat automatically switches off the heaters and maintains the temperature. The on and off mechanism of the thermostat is controlled by a thermocouple which serves as sensor to the thermostat.

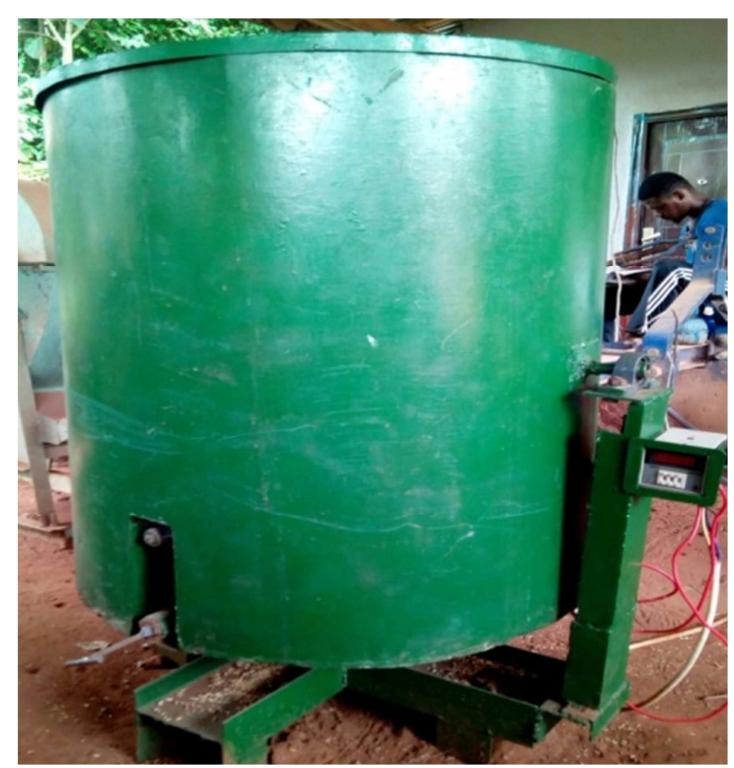

Figure 1. Automated Paddy Rice Parboiler used for the Study.

\subsection{Experimental Procedure}

The experiment was carried out to determine the effects of steeping temperature and time on the parboiling efficiency of the parboiler and the milling quality of the parboiled rice. The variety of rice used for the experiment is brown rice. The experiment was considered as a $4 \times 3 \times 3$ factorial experiment, that is, 4 levels of steeping temperature, 3 levels of steeping time and 3 replications in a Randomized Complete Block Design (RCBD) with the steeping time as the blocking factor. A constant feedrate of $20 \mathrm{~kg}$ per batch was used throughout the experiment.

\subsection{Measurements and Calculations}

\subsubsection{Parboiling Efficiency}

The parboiling efficiency is defined as the ratio of the weight of parboiled paddy rice to the total weight of the sample parboiled in single batch.

$$
\text { Parboiling Efficiency (\%) }=\frac{\text { weightofparboiledpaddyrice }}{\text { totalweightofthepaddy }} \times 100
$$

\subsubsection{Milling Quality}

The milling quality is defined as the ratio of the weight of un-cracked grains after milling to the total weight of milled rice.

$$
\text { Milling Quality }(\%)=\frac{\text { weightofuncrackedgrains }}{\text { totalweightofmilledrice }} \times 100
$$

\section{Results and Discussions}

\subsection{Statistical Analysis}

The data generated for the calculated average values of parboiling efficiency and milling quality is as shown on the Tables 1 and 2 respectively. 
Table 1. Average Value of parboiling efficiency at different steeping temperature and time.

\begin{tabular}{llll}
\hline \multirow{3}{*}{ SteepingTemperature $\left({ }^{\circ} \mathbf{C}\right)$} & \multicolumn{2}{l}{ ParboilingEfficiency(\%) } \\
\cline { 2 - 4 } & \multicolumn{2}{l}{ Steeping Time (hours) } & $\mathbf{4}$ \\
\cline { 2 - 4 } & $\mathbf{2}$ & $\mathbf{3}$ & $96.0 \pm 0.1000$ \\
60 & $95.9 \pm 0.2000$ & $97.4 \pm 0.4726$ & $97.2 \pm 0.0577$ \\
65 & $97.7 \pm 0.0577$ & $98.8 \pm 0.1155$ & $98.5 \pm 0.1155$ \\
70 & $98.5 \pm 0.0577$ & $99.7 \pm 0.1155$ & $98.0 \pm 0.0577$ \\
\hline 5
\end{tabular}

Each value is the mean of triplicate \pm standard deviation.

Table 2. Average Value of milling quality at different temperature and time.

\begin{tabular}{|c|c|c|c|}
\hline \multirow{3}{*}{ Steeping Temperature $\left({ }^{\circ} \mathrm{C}\right)$} & \multicolumn{3}{|l|}{ Millingquality(\%) } \\
\hline & \multicolumn{3}{|l|}{ Steeping Time (hours) } \\
\hline & 2 & 3 & 4 \\
\hline 60 & $90.3 \pm 0.0577$ & $91.7 \pm 0.0577$ & $90.4 \pm 0.0577$ \\
\hline 65 & $92.3 \pm 0.1155$ & $95.7 \pm 0.0000$ & $93.5 \pm 0.0577$ \\
\hline 70 & $94.6 \pm 0.0577$ & $98.5 \pm 0.0577$ & $94.7 \pm 0.1155$ \\
\hline 75 & $94.4 \pm 0.0577$ & $98.1 \pm 0.0001$ & $94.4 \pm 0.0001$ \\
\hline
\end{tabular}

Each value is the mean of triplicate \pm standard deviation.

\subsection{Analysis of Variance (ANOVA)}

The ANOVA of the effects of steeping temperature and time on the parboiling efficiency and the milling quality are as shown on the Tables 3 and 4 respectively.

Table 3. Effects of steeping temperature and time on the parboiling efficiency of the machine.

\begin{tabular}{lllcc}
\hline Source & Type III Sum of Squares & df & Mean Square & F \\
\hline Steeping Time & 13.020 & 2 & 6.510 & 558.000 \\
Steeping Temp & 30.333 & 3 & 10.111 & .002 \\
Steeping Time*Steeping Temp & .507 & 6 & .084 & .001 \\
Error & .280 & 24 & .012 & .005 \\
Total & 345082.900 & 36 & & \\
Corrected Total & 44.140 & 35 & & \\
\hline
\end{tabular}

Table 4. Effects of Steeping Temperature and Time on the Milling Quality of the Rice.

\begin{tabular}{lllll}
\hline Source & Type III Sum of Squares & df & Mean Square & F \\
\hline Steeping Time & 69.484 & 2 & 34.742 & 8338.067 \\
Steeping Temp & 151.408 & 3 & 50.469 & 12112.600 \\
Steeping Time*steeping Temp & 9.578 & 6 & 1.596 & .001 \\
Error & .100 & 24 & .004 & .002 \\
Total & 318646.250 & 36 & & \\
Corrected Total & 230.570 & 35 & & \\
\hline
\end{tabular}

The Analysis of Variance shows that the two parameters under investigation (steeping time and temperature) and the interaction between them are both significantly affecting the parboiling efficiency of the machine and the milling quality of the milled rice grains at 0.05 confidence limit.

\subsection{Duncan New Multiple Range Test (DNMRT)}

The DNMRT for the effects of steeping temperature and time on the parboiling efficiency of the machine and the milling quality of the rice grain is as shown on the Table 5 and 6 respectively. 
Table 5. DNMRT for the Effects of Steeping Temperature on the Parboiling Efficiency of the Machine and the Milling Quality of the Rice Grain.

\begin{tabular}{lll}
\hline Steeping Temperature $\left({ }^{\circ} \mathbf{C}\right)$ & Parboiling Efficiency (\%) & Milling Quality (\%) \\
\hline 60 & $96.422^{\mathrm{a}}$ & $90.789^{\mathrm{a}}$ \\
65 & $97.933^{\mathrm{b}}$ & $93.811^{\mathrm{b}}$ \\
70 & $98.889^{\mathrm{c}}$ & $95.944^{\mathrm{c}}$ \\
75 & $98.356^{\mathrm{d}}$ & $95.644^{\mathrm{d}}$ \\
\hline
\end{tabular}

*means with the same letters are not significantly difference but means with different letter are significantly different at5\% confidence level.

Table 6. DNMRT for the Effects of Steeping Time on the Parboiling Efficiency of the Machine and the Milling Quality of the Rice Grain.

\begin{tabular}{lll}
\hline Steeping Time(hours) & Parboiling Efficiency(\%) & Milling Quality(\%) \\
\hline 2 & $97.500^{\mathrm{a}}$ & $92.883^{\mathrm{a}}$ \\
3 & $98.750^{\mathrm{b}}$ & $96.000^{\mathrm{b}}$ \\
4 & $97.450^{\mathrm{a}}$ & $93.258^{\mathrm{c}}$ \\
\hline
\end{tabular}

*means with the same letters are not significantly difference but means with different letter are significantly different at $5 \%$ confidence level.

Further analysis of the result using Duncan New Multiple Range Test (DNMRT) shown on table 5 and 6 reveal that there is significant difference in the parboiling efficiency and the milling quality obtained at different steeping temperature but in the case of steeping time, the parboiling efficiency obtained at 2 and 4 hours of steeping time are the same and are significantly different from that of 3 hours. Also, the milling qualities obtained at different steeping time are significantly different at 5\% confidence level.

\subsection{Effects of Steeping Temperature and Time on the Parboiling Efficiency of the Parboiler}

The effects of steeping temperature and time on the parboiling efficiency of the parboiler are as shown on Figure
2. It can be deduce from the figure that the parboiling efficiency of the machine increases with increase in steeping temperature but tends to reduce as the temperature increased beyond $70^{\circ} \mathrm{C}$ irrespective of the steeping time.

\subsection{Effects of Steeping Temperature and Time on the Milling Quality of the Parboiler}

Figure 3 shows the effects of steeping temperature and time on the milling quality of the rice grains. The figure revealed that the milling quality of the rice grain also increases with increase in steeping temperature but tends to reduce as the temperature increased beyond $70^{\circ} \mathrm{C}$ irrespective of the steeping time.

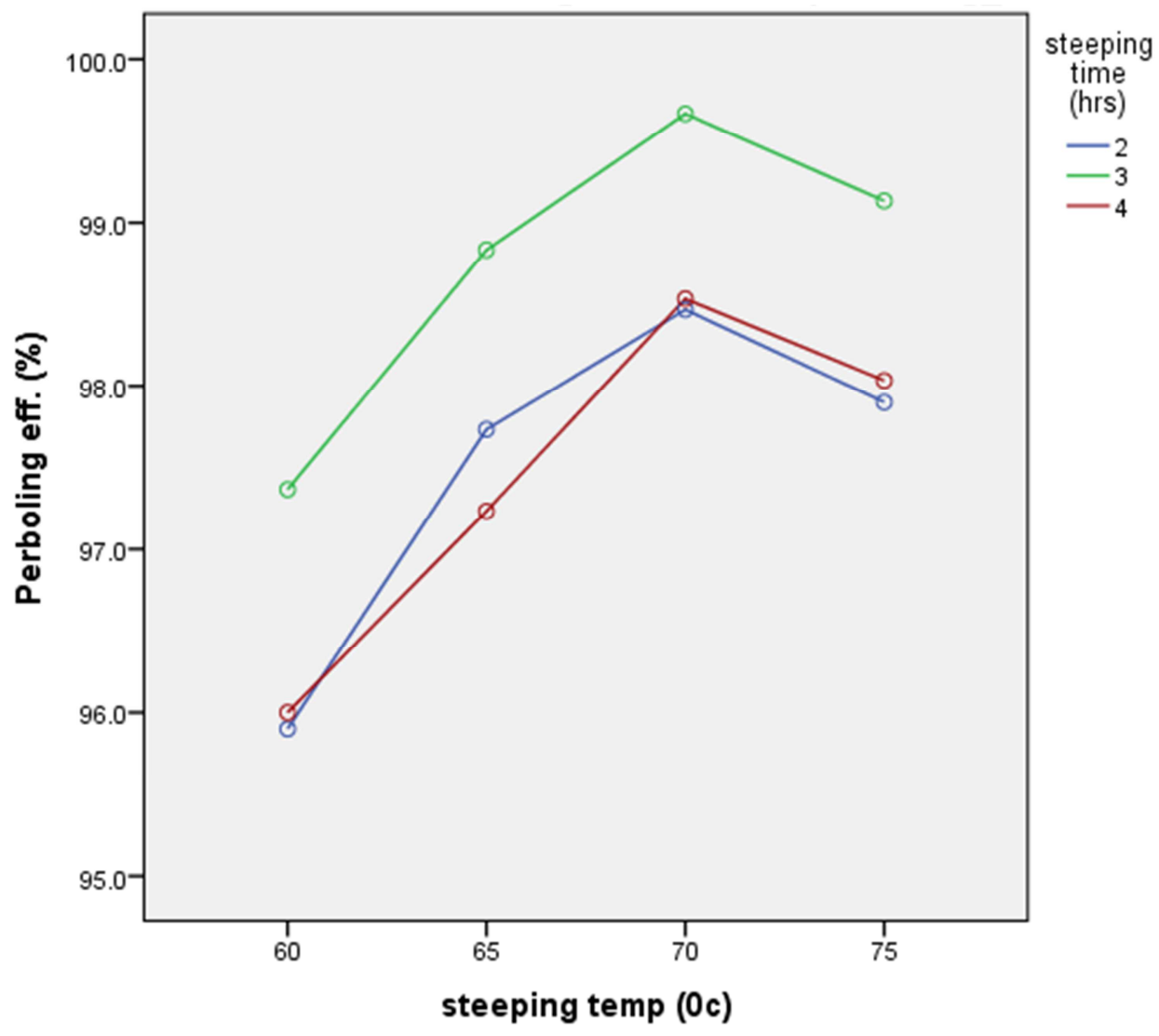

Figure 2. EffectsofSteepingTemperatureandTimeontheParboilingEfficiencyoftheParboiler. 


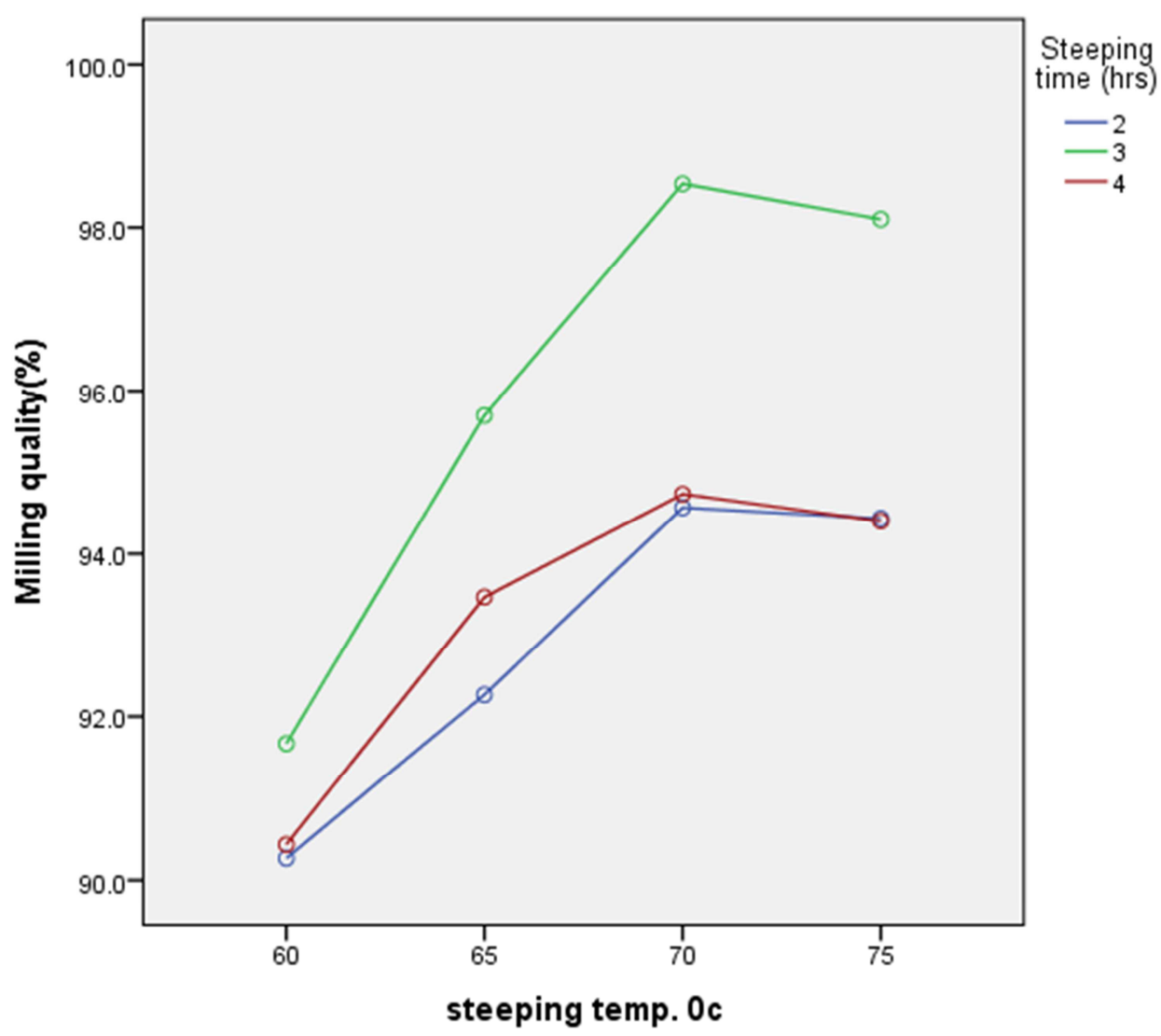

Figure 3. Effects of Steeping Temperature and Time on the Milling Quality of the Rice Grains.

\section{Conclusion}

The effects of steeping temperature and time on the parboiling efficiency and the milling quality of the parboiled paddy rice was investigated using an automated paddy rice parboiler developed in the Department of Agricultural and Bio-Environmental Engineering Technology, Auchi Polytechnic Auchi, Edo State Nigeria. The optimum parboiling efficiency of $99.7 \%$ at a steeping temperature and time of $70^{\circ} \mathrm{C}$ and $3 \mathrm{hrs}$ respectively and corresponding milling quality of $98.5 \%$ at same steeping temperature and time were obtained during the experiment.

The result of the statistical analysis shows that the steeping temperature and time has significant effects on the parboiling efficiency of the machine and milling quality of the par boiled rice grains at $5 \%$ confidence level.

\section{References}

[1] Bhattacharya K. R. 2004. Parboiling of rice Pp. 329-404 in Champagne E. T., editor., ed. Rice chemistry and technology. American Association of Cereal Chemists Inc, St. Paul, MN.

[2] Demont M., Zossou E., Rutsaert P., Ndour M., van Mele P., and Verbeke W.. 2012. Consumer valuation of improved rice parboiling technologies in Benin. Food Qual. Prefer. 23:63-70.

[3] Eliasson, A. C. (2000). Viscoelastic behaviour during the gelatinization of starch. Journal of Texture Studies, 17, 253265 .
[4] Futakuchi K., Manful J., and Takeshi S. 2013. Improving grain quality of locally produced rice $\mathrm{Pp}$. 311-323 in Wopereis M. C., editor;, Johnson D. E., editor;, Ahmadi N., editor;, Tollens E., editor;, Jalloh A., editor., eds. Realizing Africa's rice promise. CAB International, Boston, MA, USA. doi: $10.1079 / 9781845938123.0000$.

[5] Heinemann R. J. B., Behrens J. H., and Lanfer-Marquez U. M. 2006. A study on the acceptability and consumer attitude towards parboiled rice. Int. J. Food Sci. Technol. 41:627-634.

[6] Manful, J. T., Abbey L. D., and Coker R. D.. 2009. Effect of artisanal parboiling methods on milling yield and cooked rice textural characteristics. J. Food Qual. 32:725-734.

[7] Ndindeng, S. A., Mapiemfu D. L., Fantong W., Nchinda V. P., Ambang Z., and Manful J. T.. 2014. Postharvest adaptation strategies to the effects of temperature variations and farmermiller practices on the physical quality of rice in Cameroon. Am. J. Climate Change 3:178-192.

[8] Odenigbo, A. M., Ndindeng S. A., Nwankpa C. A., Woin N., and Ngadi M.. 2013. In-vitro starch digest-ibility and nutritional composition of improved rice varieties from Cameroun. Eur. J. Nutr. Food Saf. 3:134-145.

[9] Prom-U-thai C., Rerkasem B., Cakmak I., and Huang L. 2010. Zinc fortification of whole rice grain through parboiling process. Food Chem. 120:858-863.

[10] Rao S. R., and Juliano B. O. 1970. Effect of parboiling on some physicochemical properties of rice. J. Agric. Food Chem. 18:289-294. 